\title{
ANATOMICAL VARIATION OF RADIAL WRIST EXTENSOR MUSCLES: A STUDY IN CADAVERS
}

Soubhagya Ranjan Nayak, Ashwin Krishnamurthy, Latha Venkatraya Prabhu, Rajalakshmi Rai, Anu Vinod Ranade, Sampath Madhyastha

Nayak SR, Krishnamurthy A, Prabhu LV, Rai R, Ranade AV, Madhyastha S. Anatomical Variation of Radial Wrist Extensor Muscles: A Study in Cadavers. Clinics. 2008;63(1):85-90.

OBJECTIVE: The tendons of the extensor carpi radialis longus and brevis muscles are quite useful in tendon transfer, such as in correction of finger clawing and restoration of thumb opposition. Knowledge of additional radial wrist extensor muscle bellies with independent tendons is useful in the above-mentioned surgical procedures.

METHODS: The skin, subcutaneous tissue, and antebrachial fascia of 48 (24 on the right side and 24 on left side) male upper limb forearms were dissected. The following aspects were then analyzed: (a) the presence of additional muscle bellies of radial wrist extensors, (b) the origin and insertion of the additional muscle, and (c) measurements of the muscle bellies and their tendons. RESULTS: Five out of 48 upper limbs (10.41\%) had additional radial wrist extensors; this occurred in 3 out of 24 left upper limbs $(12.5 \%)$ and 2 out of 24 right upper limbs (8.3\%). In one of the right upper limbs, two additional muscles were found. The length and width of each additional muscle belly and its tendon ranged between $2-15 \mathrm{~cm}$ by $0.35-6.4 \mathrm{~cm}$ and $2.8-20.8 \mathrm{~cm}$ by 0.2 $0.5 \mathrm{~cm}$, respectively. The additional radial wrist extensor tendons in our study basically originated either from the extensor carpi radialis longus or brevis muscles and were inserted at the base of the $2^{\text {nd }}$ or $3^{\text {rd }}$ metacarpal bone.

CONCLUSION: The present study will inform surgeons about the different varieties of additional radial wrist extensors and the frequency of their occurrence.

KEYWORDS: Extensor carpi radialis longus. Extensor carpi radialis brevis. Occurrence. Tendon transfer. Clinical significance.

\section{INTRODUCTION}

The extensor carpi radialis longus (ECRL) and brevis (ECRB) are two muscles that belong to the radial wrist extensors (RWE). The ECRL arises from the distal third of the lateral supracondylar ridge of the humerus and the adjacent portion of the lateral intermuscular septum, while the ECRB arises from the front of the lateral epicondyle of the humerus and the fascia covering the common extensor origin. Both of these radial extensors run through the $2^{\text {nd }}$ compartment of the extensor retinaculum; the ECRL is inserted into the base of the second metacarpal bone, and

Kasturba Medical College, Department of Anatomy, CBS, Bejai Mangalore - Karnataka, India.

ranjanbhatana@gmail.com

Received for publication on September 11, 2007.

Accepted for publication on October 05, 2007. the ECRB is inserted into the third ${ }^{1}$. According to a study conducted by Caetano et al. (2004) the most common pattern of the ECRL and ECRB was one muscle and one tendon each. Out of 60 upper limbs, they found additional RWE tendons in only 3 hands (all in relation to the ECRL tendon). The presence of an accessory tendon connecting the tendons of the ECRL and ECRB was identified in 4 dissected hands ${ }^{2}$. Albright and Linburg (1978) found that $26 \%$ of upper limbs had an accessory tendon joining the tendons of the ECRL and ECRB. The occurrence rate of an additional RWE was $24 \%$ in the same study (42 out of 173 upper limbs), and the additional muscles originated from either the ECRL or ECRB muscles ${ }^{3}$.

The importance of tendon transfer using RWE in opponensplasty and correction of finger clawing has been reported by several authors ${ }^{4-6}$. Cooney et al. (1984) studied various muscles to achieve effective tendon transfer 
for median nerve palsy. They found the ECRL muscle to be one of the best at approximating the force and motion required to restore lost thumb flexion, full thumb opposition and strength both in high and low median nerve palsy ${ }^{4}$. Baek et al. (1999) used either the ECRL or ECRB to restore opposition of the thumb. Out of the 11 transfers they performed, 10 gave excellent results ${ }^{5}$. Studies on the RWE were undertaken by various authors ${ }^{3,7-13}$. Wood (1988) described the presence of additional RWE that could be used successfully for thumb opposition by motoring the flexor pollicis longus and extensor pollicis longus of the thumb ${ }^{12}$. The additional RWE tendons in our study were inserted into the dorsal digital expansion of the index finger (DDEIF), the base of the second metacarpal bone (B2M), the base of the third metacarpal bone (B3M), the base of both the second and third metacarpal bones (B2/3M) and the ECRB. In the past decade, unusual forms of RWE have been mentioned in the literature $^{14-17}$. The purpose of the present study was to identify the occurrence and various forms of additional RWE. This knowledge should help surgeons to reduce their error in diagnosis and treatment in and around the radial wrist extensors when additional muscle bellies and their tendons exist. This work was motivated by the paucity of literature regarding the origin of additional RWE and their immense importance in tendon transfers.

\section{MATERIALS AND METHODS}

During routine cadaver dissection in the Department of Anatomy, Kasturba Medical College, Mangalore, India, we dissected 24 formalin-embalmed male (47- 75) upper limbs during the academic years, 2004-2005 and 2005-2006. The skin, superficial fascia, and antebrachial fascia from the lateral aspect of the forearms were then excised, and the individual RWE were studied for the presence of any additional muscles. When identified, anatomical description of the additional RWE was achieved by measuring its length, width and attachments. Measurements of the additional muscles and their tendons were done with the help of a Vernier caliper in centimeters $(\mathrm{cm})$.

\section{RESULTS}

Out of 48 upper limbs studied, five (10.41\%) had additional RWE (Fig 1, 2, 3a, 3b, 4, 5). Three out of 24 left upper limbs (12.5\%) and 2 out of 24 right upper limbs (8.3\%) had additional RWE. In one of the right upper limbs, there were two additional muscles found (Fig 3a, $3 \mathrm{~b})$. The length and width of each additional muscle belly and its tendon are mentioned in Table 1.

\section{Anatomical variation 1}

The additional muscle took its origin from the ECRB and then passed between the ECRB and extensor digitorum communis (EDC) in the forearm. Its tendon ran below the extensor retinaculum, along with the tendons of the EDC and extensor indicis in the fourth dorsal osseofibrous tunnel, and was inserted into the DDEIF. The tendon of the EDC to the IF was absent in the above case (Fig 1). The length and width of the additional muscle belly and tendon were $11.4 \mathrm{~cm}$ by $1.3 \mathrm{~cm}$ and $15 \mathrm{~cm}$ by $0.25 \mathrm{~cm}$, respectively.

\section{Anatomical variation 2}

The additional muscle took a tendinous origin from the lateral epicondyle of the humerus (LEH), between the origin of the ECRB and EDC muscles, and was on the medial side of the ECRL and ECRB muscles. Fifteen centimeters from its origin, the muscle divided into two tendons below the abductor pollicis longus (APL) and extensor pollicis brevis (EPB) muscles. The upper tendon then traveled side by side with the tendon of the ECRB muscle and passed through the second dorsal osseofibrous tunnel to be inserted into the $\mathrm{B} 2 \mathrm{M}$, medial to the insertion of the ECRB tendon. The lower tendon crossed over the ECRL tendon and also passed through the second dorsal osseofibrous tunnel to be inserted into the radial side of the B3M, above the insertion of ECRL (Fig 2). The length and width of the additional muscle bellies and tendons were $15 \mathrm{~cm}$ by $6.4 \mathrm{~cm}$ for the upper and lower muscle bellies, $10.2 \mathrm{~cm}$ by $0.5 \mathrm{~cm}$ for the upper tendon and $10.5 \mathrm{~cm}$ by $0.45 \mathrm{~cm}$ for the lower tendon.

\section{Anatomical variation 3a}

The additional muscle took its origin from the ECRB, between the ECRB and ECRL muscles. The tendon of the additional muscle was placed between the ECRB and ECRL tendons in the second dorsal osseofibrous tunnel and was inserted between the B2/3M (Fig 3a). The length and width of the additional muscle belly and tendon were $3 \mathrm{~cm}$ by $1.1 \mathrm{~cm}$ and $19.4 \mathrm{~cm}$ by $0.3 \mathrm{~cm}$, respectively.

\section{Anatomical variation 3b}

The additional muscle took its origin from the undersurfaces of both the ECRL (medial) and brachioradialis (BR), then formed a common muscle and was inserted on the upper surface of the ECRB tendon (Fig $3 b)$. The length of the additional muscle belly was $2 \mathrm{~cm}$ 


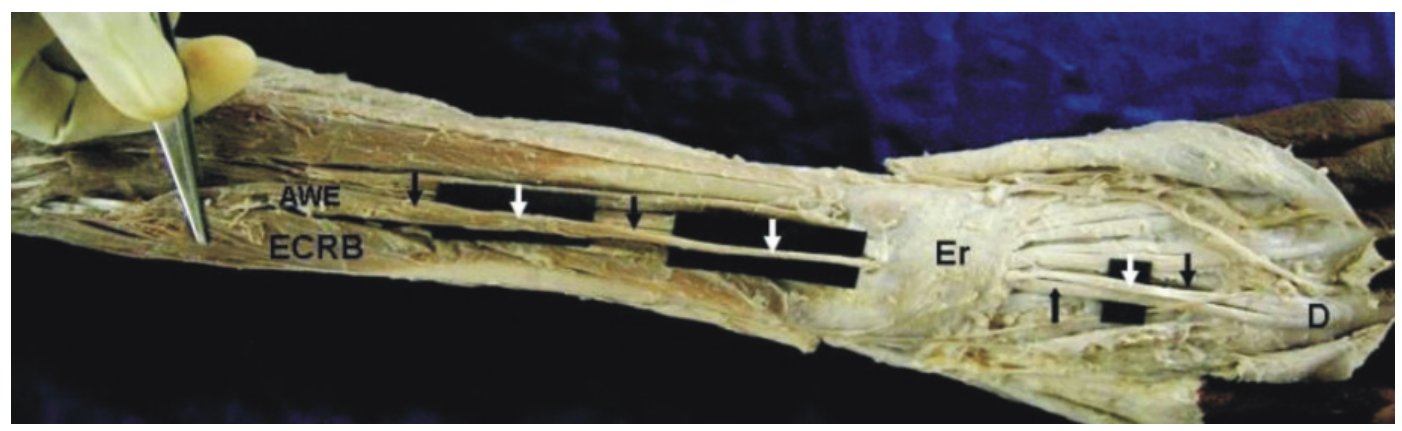

Figure 1 - Extensor compartment of the left forearm and hand region. AWE, additional wrist extensor; D, dorsal digital expansion for the index finger; ECRB, extensor carpi radialis brevis; Er, extensor retinaculum. The course of the additional muscle is shown by the downward facing arrows. The upward facing arrow indicates the tendon of extensor indicis muscle.

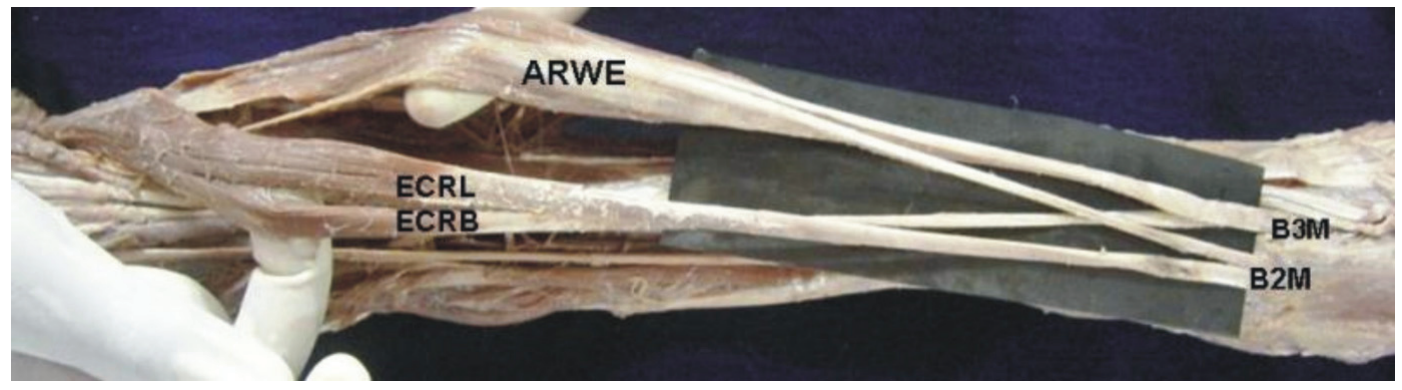

Figure 2 - Extensor compartment of the left forearm and hand region. ARWE, additional radial wrist extensor; B2M, base of the second metacarpal bone; $\mathrm{B} 3 \mathrm{M}$, base of the third metacarpal bone; ECRL, extensor carpi radialis longus.

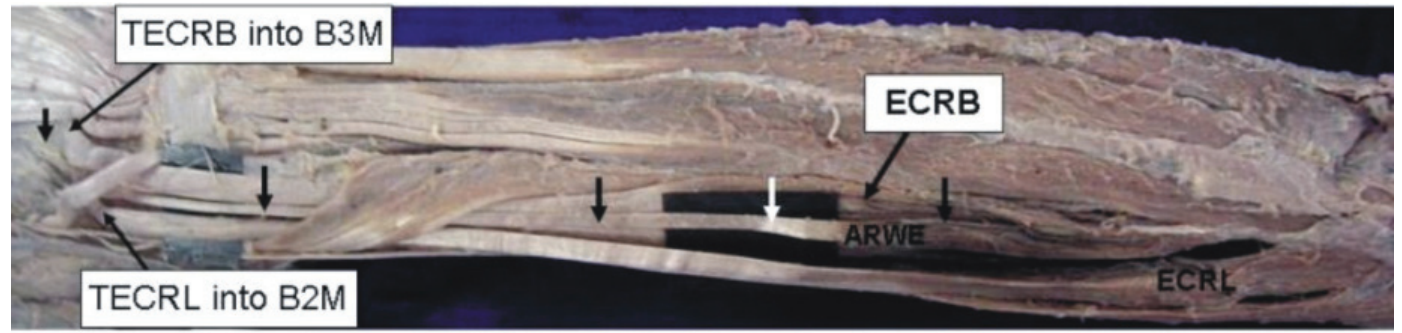

Figure 3a - Extensor compartment of the right forearm and hand region. TECRB into B3M, tendon of extensor carpi radialis brevis inserted into the base of the third metacarpal bone; TECRL into B2M, tendon of extensor carpi radialis longus inserted into the base of the second metacarpal bone. Note that the downward arrows indicate the course of the additional muscle.

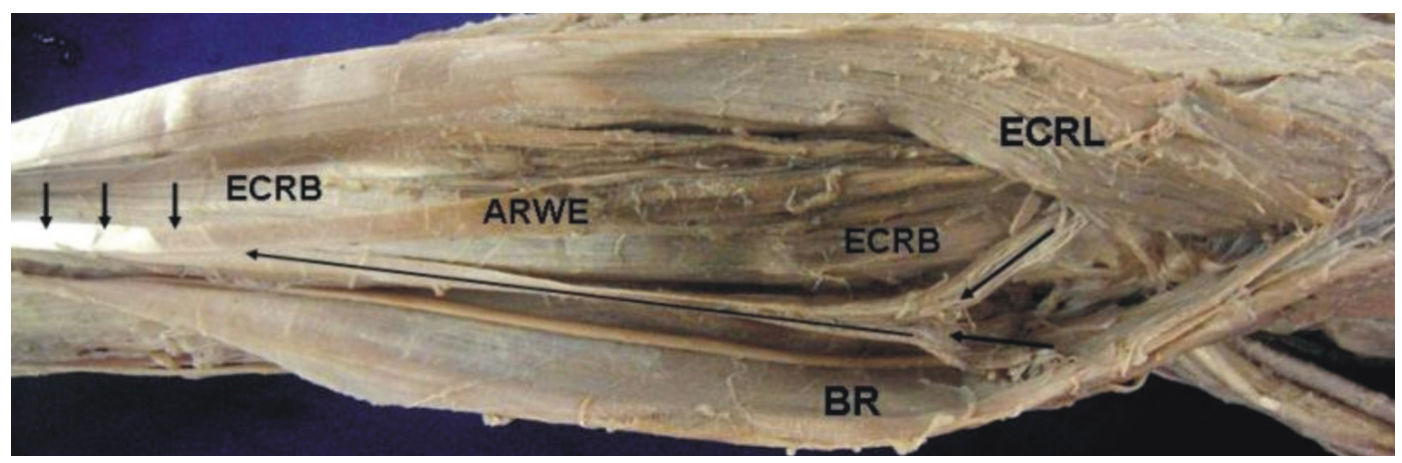

Figure 3b - Superior-lateral view of the right forearm region. ARWE, additional radial wrist extensor. The downward arrows indicate its course, already shown in figure 3a. BR, brachioradialis. Note the additional muscles arising both from the BR (lateral) and ECRL (medial), joining to form a single muscle on its way to be inserted into the tendon of the ECRB. 


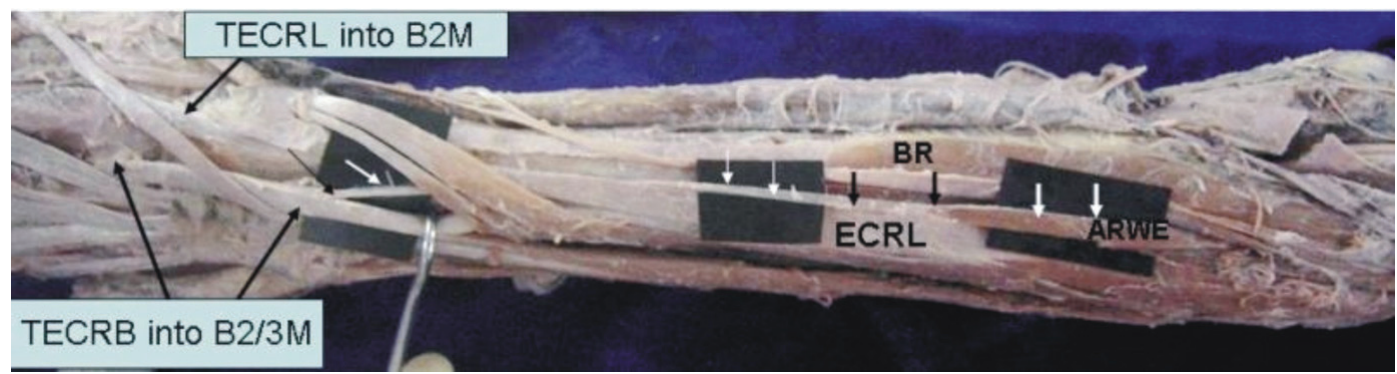

Figure 4 - Extensor compartment of the left forearm and hand region. TECRB into B2/3M, tendon of extensor carpi radialis brevis inserted into the base of the second and third metacarpal bones; TECRL into $\mathrm{B} 2 \mathrm{M}$, tendon of extensor carpi radialis longus inserted into the base of the second metacarpal bone. Note that the downward arrows indicate the course of the additional muscle. The muscle was inserted into the tendon of the ECRB.

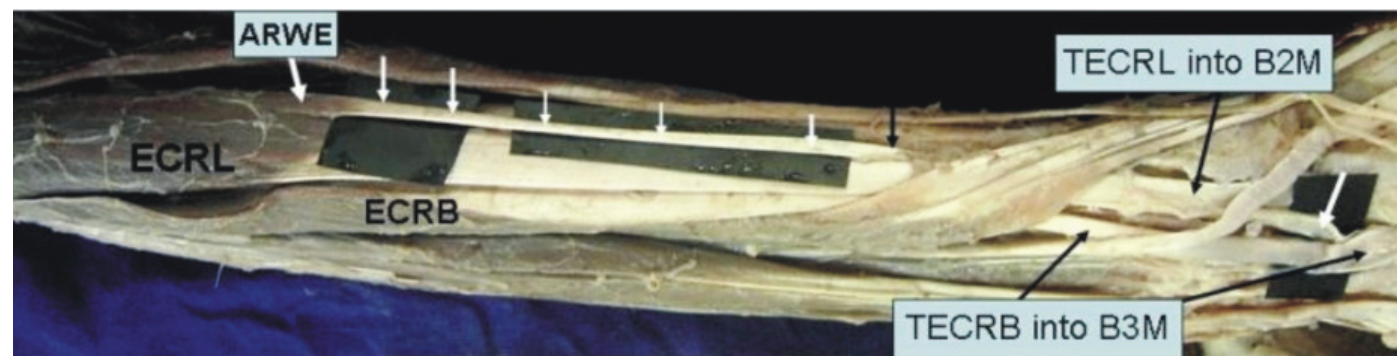

Figure 5 - Extensor compartment of the right forearm and hand region. TECRB into B3M, tendon of extensor carpi radialis brevis inserted into the base of the third metacarpal bone; TECRL into B2M, tendon of extensor carpi radialis longus inserted into the base of the second metacarpal bone. Note the downward arrows, which indicate the course of the additional muscle.

Table 1 - Origin, insertion and measurements of the additional radial wrist extensors (ARWEs).

\begin{tabular}{lccccccc}
\hline No. & Side & Origin & Insertion & Muscle Length & Muscle Width & Tendon Length & Tendon Width \\
\hline 1 & Left & ECRB & DDEIF & $11.4 \mathrm{~cm}$ & $1.3 \mathrm{~cm}$ & $15 \mathrm{~cm}$ & $0.25 \mathrm{~cm}$ \\
2 & Left & LEH & UT=B2M & $15 \mathrm{~cm}$ & $6.4 \mathrm{~cm}$ & $10.2 \mathrm{~cm}$ & $0.5 \mathrm{~cm}$ \\
& & & LT=B3M & $15 \mathrm{~cm}$ & $6.4 \mathrm{~cm}$ & $10.5 \mathrm{~cm}$ & $0.45 \mathrm{~cm}$ \\
$3 \mathrm{a}$ & Right & ECRB & B $2 / 3 \mathrm{M}$ & $3 \mathrm{~cm}$ & $1.1 \mathrm{~cm}$ & $19.4 \mathrm{~cm}$ & $0.3 \mathrm{~cm}$ \\
$3 \mathrm{~b}$ & Right & BR \& ECRL & ECRB & BR=2cm & $0.5 \mathrm{~cm}$ & $2.8 \mathrm{~cm}$ & $0.4 \mathrm{~cm}$ \\
& & & ECRL $=2.5 \mathrm{~cm}$ & $0.6 \mathrm{~cm}$ & $0.35 \mathrm{~cm}$ & $12.8 \mathrm{~cm}$ & $0.2 \mathrm{~cm}$ \\
4 & Left & ECRL & ECRB & $6.2 \mathrm{~cm}$ & $0.4 \mathrm{~cm}$ & $20.8 \mathrm{~cm}$ & $0.3 \mathrm{~cm}$ \\
5 & Right & ECRL & B3M & $2 \mathrm{~cm}$ & & \\
\hline
\end{tabular}

ECRB, extensor carpi radialis brevis; DDEIF, dorsal digital expansion for the index finger; LEH, lateral epicondyle of humerus; UT, upper tendon; B2M, base of the second metacarpal bone; LT, lower tendon; B3M, base of the third metacarpal bone; B2/3M, to the base of the both second and third metacarpal bones; BR, brachioradialis; ECRL, extensor carpi radialis longus.

from the BR and $2.5 \mathrm{~cm}$ from the ECRL, while the width was $0.5 \mathrm{~cm}$ from the BR and $0.6 \mathrm{~cm}$ from the ERCL. The length and width of the additional muscle tendon were 2.8 $\mathrm{cm}$ and $0.4 \mathrm{~cm}$, respectively.

\section{Anatomical variation 4}

The additional muscle took its origin from the radial side of the ECRL, and the tendon passed below the APL and EPB muscles to be inserted on the undersurface of the ECRB tendon (Fig 4). The length and width of the additional muscle belly and tendon were $6.2 \mathrm{~cm}$ by $0.35 \mathrm{~cm}$ and $12.8 \mathrm{~cm}$ by $0.2 \mathrm{~cm}$, respectively.

\section{Anatomical variation 5}

The additional muscle took its origin from the radial side of the ECRL, and the tendon passed below the second dorsal osseofibrous tunnel to be inserted into the B3M (Fig 5). The length and width of the additional muscle belly and tendon were $2 \mathrm{~cm}$ by $0.4 \mathrm{~cm}$ and $20.8 \mathrm{~cm}$ by $0.3 \mathrm{~cm}$, respectively.

\section{DISCUSSION}

The extensor carpi radialis intermedius (ECRI) and extensor carpi radialis accessories (ECRA) are the two pre- 
viously described variants of additional RWE in the antebrachial region (Wood, 1867 as cited by Wood, 1988; Wood, 1867 as cited by MacAlister, 1871) $)^{7,8,12}$. In the present study, we found that the additional RWE originated from the ECRL in three cases, from the ECRB in two cases, and from the BR and $\mathrm{LEH}$ in one case each. The additional RWE tendons inserted into various sites on the dorsal aspect of the hand, namely the DDEIF, B2M, B3M, B2/3M and the tendon of the ECRB muscle. The length of the additional RWE varied from two to $15 \mathrm{~cm}$, and the muscle width also varied from 0.35 to $6.4 \mathrm{~cm}$. The tendon length of the additional RWE varied from 2.8 to $20.8 \mathrm{~cm}$, and the tendon width also varied from 0.2 to $0.5 \mathrm{~cm}$. All of the additional RWE tendons were large enough to perform a tendon transfer.

Wood (1988) $)^{12}$ examined 312 upper limbs and found 39 (12.5\%) ECRI, of which $32(10.25 \%)$ tendons were suitable for muscle transfer and 7 (2.24\%) were unacceptable. In the present study, we identified the occurrence of additional RWE in $10.41 \%$ of upper limbs, which was similar to the study by Wood (1988) ${ }^{12}$. Albright and Linburg (1978) ${ }^{3}$ studied 173 upper limb specimens, of which they found 42 limbs (24\%) with an extra muscle that originated either from the ECRL or ECRB muscle. In the present study we found additional RWE in $10.41 \%$ of upper limbs in comparison to the $24 \%$ found by Linburg $(1978)^{3}$. Although the origins of additional RWE were mainly from the ECRL and ECRB, we also noted origins from the LEH and BR.
Baek et al. (1999) $)^{5}$ used ECRL and ECRB tendons instead of the flexor digitorum profundus (FDP) tendon to restore thumb opposition. To their surprise, they found that the RWE tendons produced excellent results. Malaviya $(2003)^{6}$ used split ECRL tendons to correct finger clawing and was able to reduce operating time, muscle herniation and scarring at the donor site. Cooney et al. (1984) ${ }^{4}$ studied forearm and hand muscle volume, mean fiber length, and cross-sectional area for tendon transfers done for opposition of thumb and found that $60 \%$ of ECRL tendons were effective. Sabapathy et al. $(2005)^{18}$ found that ECRL tendon transfer for restoration of finger flexion in patients with flexor muscle loss was a simple alternative, which should be considered when possible.

The present study on RWE supplements knowledge about the various forms of additional RWE and their occurrence. Furthermore, the morphometric measurements of the additional muscles and their tendons will help surgeons performing tendon transfer in the antebrachial and carpal region.

\section{CONCLUSION}

In the present study, we found five anatomical variations in the radial wrist extensor muscles. The length and width measurements of the anatomical variations were considered sufficient to warrant their use for tendon transfers.

\section{REFERENCES}

1. William PL, Warwick R, Dyson M, Bannister LH. The muscles of the fore arm. Gray's Anatomy. $37^{\text {th }}$ ed. Edinburgh: Churchill Livingstone; 1989. p. 622.

2. Caetano FM, Albertoni MW, Caetano BE, Perez MR. Anatomical study of insertions of the extensor carpi radialis longus and brevis. Int $\mathbf{J}$ Morphol. 2004;22:245-51.

3. Albright JA, Linburg RM. Common variations of the radial wrist extensors. J Hand Surg. 1978; 3:134-8.

4. Cooney WP, Linscheid RL, An KN. Opposition of the thumb: an anatomical and biomechanical study of tendon transfers. J Hand Surg. 1984;9:777-86.

5. Baek GH, Jung JM, Yoo WJ, Chung MS. Transfer of extensor carpi radialis longus or brevis for opponensplasty. J Hand Surg. 1999;24:503.

6. Malaviya GN. Radial half of extensor carpi radialis longus tendon as graft to elongate muscle tendon unit for correction of finger clawing. Plast Reconstr Surg. 2003;111:1914-7.
7. Wood J. Variations in human myology observed during the winter session of 1866-67 at King's College London. Proc. R. Soc. $1867 ; 15: 518-46$.

8. MacAlister A. Additional observations on muscular anomalies in human anatomy with a catalogue of the principal muscular variations hitherto published (third series). Trans. R. Ir. Acad. 1871;25:101-2.

9. Frohse F, Frankel M. Die Muskeln des menschlichen Armes. In: Bardeleben, Kv. (Ed.), Handbuchs der Anatomie des Menschen. Fischer, Jena, 1908. p. 160-1.

10. Valentin P. Extrinsic muscles of the hand and wrist: an introduction. In: Tubiana, R. (Ed.), The Hand. Philadelphia: WB Saunders Company; 1981. p. 243.

11. Kaplan EB, Spinner M. Important muscular variations of the hand and forearm. In: Spinner, M. (Ed.), Kaplan's Functional and Surgical Anatomy of the Hand., Philadelphia: JB Lippincott Company; 1984. p. $340-1$

12. Wood VE. The extensor carpi radialis intermedius tendon. J Hand Surg. 1988;13:242-5. 
13. Yoshida Y. Anatomical studies on the extensor carpi radialis longus and brevis muscles in Japanese. Okajimas Folia Anat Jpn. 1994;71:127-35.

14. Melling M, Steindl M, Wilde J, Karimian-Teherani D. An anatomical variant of the extensor carpi radialis brevis muscle. Wien Klin Wochenschr. 2001;113:960-3.

15. Mitsuyasu H, Yoshida R, Shah M, Patterson RM, Viegas SF. Unusual variant of the extensor carpi radialis brevis muscle: a case report. Clin Anat. 2004;17:61-3.
16. Hong MK, Hong MK. An uncommon form of the rare extensor carpi radialis accessorius. Ann Anat. 2005;187:89-92.

17. Nayak SR, Madhan Kumar SJ, Krishnamurthy A, Prabhu LV, Ranade AV, Rai R et al. An additional radial wrist extensor and its clinical significance. Ann Anat. 2007;189:283-6.

18. Sabapathy SR, Gowda DK, Ranade AB, Venkatramani H, Sebastin SJ. Functional outcome of extensor carpi radialis longus transfer for finger flexion in posttraumatic flexor muscle loss. J Hand Surg. 2005;30:26772. 